\title{
Classical complication of infective endocarditis: ruptured, large mycotic cerebral aneurysm
}

\author{
Nitinan Chimparlee, ${ }^{1}$ Pakrit Jittapiromsak, ${ }^{2}$ Jarturon Tantivatana, ${ }^{3}$ \\ Pairoj Chattranukulchai ${ }^{4}$
}

'Department of Biochemistry, Faculty of Medicine, Chulalongkorn University, Bangkok, Thailand 2Division of Neurosurgery, Department of Surgery, Faculty of Medicine, Chulalongkorn University, Bangkok, Thailand ${ }^{3}$ Department of Radiology, Faculty of Medicine, Chulalongkorn University, Bangkok, Thailand ${ }^{4}$ Division of Cardiology, Department of Medicine, Faculty of Medicine, Chulalongkorn University, Cardiac Center, King Chulalongkorn Memorial Hospital, Bangkok, Thailand

\section{Correspondence to} Dr Pairoj Chattranukulchai, pairoj.md@gmail.com

Accepted 11 March 2014

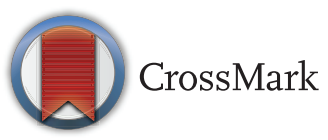

To cite: Chimparlee $\mathrm{N}$ Jittapiromsak $\mathrm{P}$,

Tantivatana J, et al. BMJ Case Rep Published online: [please include Day Month Year] doi:10.1136/bcr-2013202275

\section{DESCRIPTION}

A 16-year-old female patient who had a history of asymptomatic valvular heart disease came to the emergency department with sudden onset of severe headache. She had noticed a low-grade fever for 2 months. On examination, she was fully conscious but in distress. Pansystolic murmur grade III/VI was noted at the apex. Neurological examination showed positive meningeal irritation signs without focal neurological deficit. Axial non-contrast cerebral CT revealed early extravasation of blood into the subarachnoid space at the basal cistern (figure 1A, arrow head). Ruptured, large, $1.8 \mathrm{~cm}$ aneurysm at the tip of the basilar artery was identified from cerebral angiography (figure 1B,C, arrows). Transthoracic echocardiography discovered a large, $3 \mathrm{~cm}$, highly mobile vegetation attaching the mitral valve (figure $2 \mathrm{~A}-\mathrm{C}$, arrows) with moderate mitral regurgitation. Streptococcus viridians bacteraemia was confirmed by the consecutive blood cultures. She was finally diagnosed as having subacute infective endocarditis (IE) complicated by a ruptured mycotic aneurysm, an abnormal focal dilation of the infected arterial wall due to bacteraemia or septic embolisation. Antimicrobial treatment followed by endovascular coil embolisation of the aneurysm was successfully carried out. The patient underwent mitral valve replacement soon with an uneventful postoperative period.

Neurological complication is a common manifestation of left-sided native valve IE. It can develop in $25 \%$ of cases and lead to a higher mortality rate, especially in vegetation size $\geq 3 \mathrm{~cm}$, Staphylococcus aureus infection, mitral valve involvement and history of anticoagulant use. Septic embolisation may cause ischaemic stroke, mycotic aneurysm, intracranial haemorrhage,
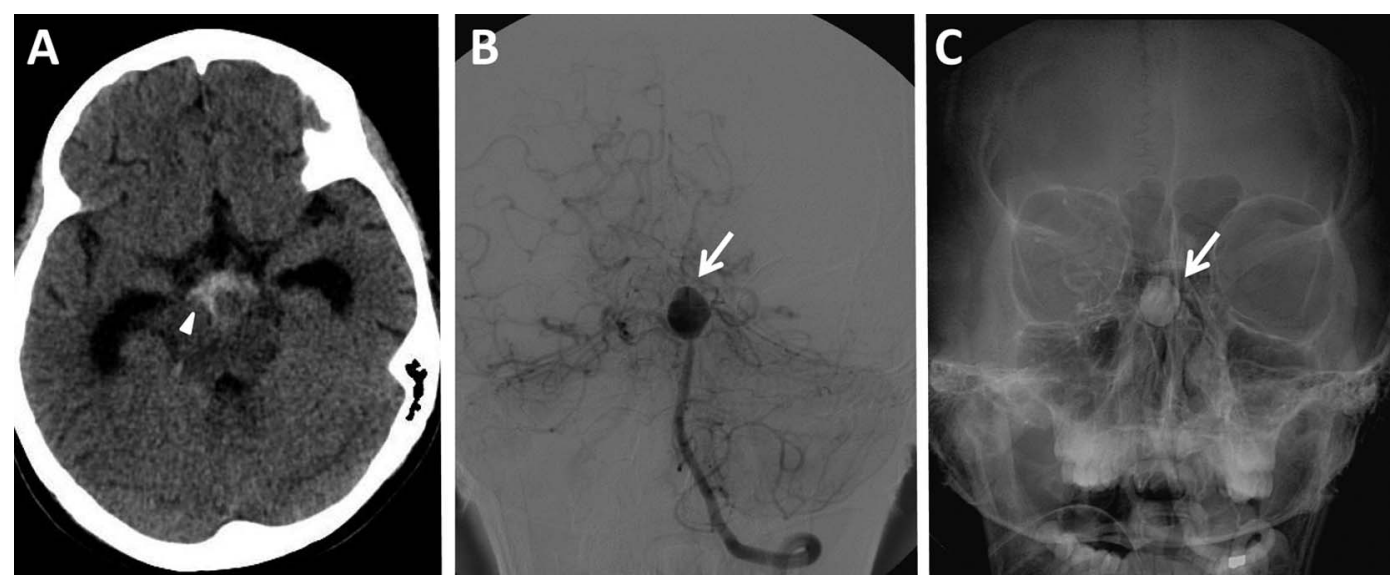

Figure 1 Axial non-contrast cerebral CT revealed early extravasation of blood into the subarachnoid space at the basal cistern ( $A$, arrow head). Ruptured, large, $1.8 \mathrm{~cm}$ aneurysm at the tip of the basilar artery was identified from cerebral angiography ( $B$ and $C$, arrows).
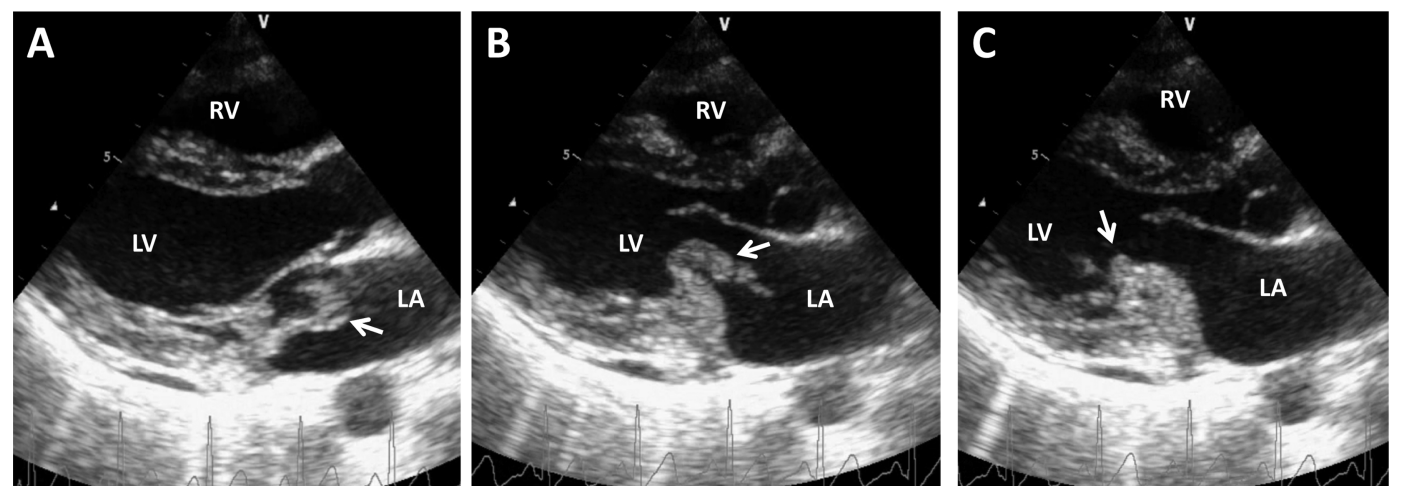

Figure 2 Transthoracic echocardiography discovered a large, $3 \mathrm{~cm}$, highly mobile vegetation attaching to the mitral valve (arrows). Multiphasic imaging throughout the cardiac cycle is shown; during systole (A), early diastole (B) and late diastole (C). LV, left ventricle; RV, right ventricle; LA, left atrium. 
meningitis and brain abscess. ${ }^{1} 2$ Early antimicrobial treatment can reduce the risk of this catastrophic neurological complication. ${ }^{1}$ Unruptured mycotic aneurysm may improve solely with antimicrobial treatment; however, patients with a ruptured aneurysm should undergo urgent interventions such as embolisation or surgery. ${ }^{3}$

\section{Learning points}

- Neurological complication is a common manifestation of left-sided native valve infective endocarditis.

- Septic embolisation may cause ischaemic stroke, mycotic aneurysm, intracranial haemorrhage, meningitis and brain abscess.

- Early antimicrobial treatment can reduce the risk of these catastrophic neurological complications.
Contributors NC and PC wrote the manuscript. JT and PJ reviewed and revised the manuscript.

Competing interests None.

Patient consent Obtained.

Provenance and peer review Not commissioned; externally peer reviewed.

\section{REFERENCES}

1 García-Cabrera E, Fernández-Hidalgo N, Almirante B, et al. Neurologic complications of infective endocarditis: risk factors, outcome, and impact of cardiac surgery: a multicenter observational study. Circulation 2013;127: 2272-84.

2 Pruitt AA. Neurologic complications of infective endocarditis. Curr Treat Options Neurol 2013;15:465-76.

3 Kannoth S, Thomas SV. Intracranial microbial aneurysm (infectious aneurysm): current options for diagnosis and management. Neurocrit Care 2009;11: $120-9$.

Copyright 2014 BMJ Publishing Group. All rights reserved. For permission to reuse any of this content visit http://group.bmj.com/group/rights-licensing/permissions.

BMJ Case Report Fellows may re-use this article for personal use and teaching without any further permission.

Become a Fellow of BMJ Case Reports today and you can:

- Submit as many cases as you like

- Enjoy fast sympathetic peer review and rapid publication of accepted articles

- Access all the published articles

- Re-use any of the published material for personal use and teaching without further permission

For information on Institutional Fellowships contact consortiasales@bmjgroup.com

Visit casereports.bmj.com for more articles like this and to become a Fellow 